\title{
Circulating miR-17-5p and miR-20a: Molecular markers for gastric cancer
}

\author{
MEI WANG ${ }^{1}$, HONGBING GU $^{1,2}$, SHENG WANG $^{2}$, HUI QIAN ${ }^{1}$, WEI ZHU ${ }^{1}$, LING ZHANG $^{1}$, \\ CHONGHUI ZHAO ${ }^{1}$, YANG TAO ${ }^{1,2}$ and WENRONG XU ${ }^{1}$ \\ ${ }^{1}$ Department of Clinical Laboratory Medicine, School of Medical Science and Laboratory Medicine, \\ Jiangsu University, Jiangsu 212013; ${ }^{2}$ Department of Clinical Laboratory Medicine, \\ The Affiliated Hospital of Jiangsu University, Jiangsu 212001, P.R. China
}

Received October 3, 2011; Accepted January 3, 2012

DOI: $10.3892 / \mathrm{mmr} .2012 .828$

\begin{abstract}
Circulating miR-17-5p and miR-20a (miR-17-5p/20a) have been demonstrated to be elevated in the plasma of gastric cancer patients. However, the clinical significance of the circulating levels of these microRNAs (miRNAs), the predictive power for prognosis and application for monitoring of chemotherapeutic effects remain unclear. To this end, we measured plasma miR-17-5p/20a levels in unpaired pre-operative $(\mathrm{n}=65)$, post-operative $(n=16)$ and relapse $(n=6)$ gastric cancer patient groups. The 3-year overall survival rate for the unpaired pre-operative patients was recorded. The circulating levels of miR-17-5p/20a were also tested in paired pre-operative and post-operative plasma from 14 gastric cancer patients. We found that the concentrations of miR-17-5p/20a were significantly associated with the differentiation status and TNM stages of gastric cancer. The miRNA levels in the different groups reflected pathological tumor progression. Kaplan-Meier curve analysis revealed that high expression levels of miR-17-5p/20a were significantly correlated with poor overall survival. Cox regression analysis demonstrated that the level of plasma miR-20a was an independent risk predictor for prognosis. An in vivo mouse tumor model was established and antagomirs against miR-17-5p/20a were applied as chemotherapeutics to perform tumor treatment. An assay of serum miR-17-5p/20a levels showed that the levels of serum miRNAs were notably reduced in post-treated mice with tumor volume regression. Taken together, the levels of circulating miR-17-5p/20a may be a promising non-invasive molecular marker for pathological progression, prediction of prognosis and monitoring of chemotherapeutic effects for gastric cancer.
\end{abstract}

Correspondence to: Dr Wenrong Xu, Department of Clinical Laboratory Medicine, School of Medical Science and Laboratory Medicine, Jiangsu University, 301 Xuefu Road, Zhenjiang, Jiangsu 212013, P.R. China

E-mail: icls@ujs.edu.cn

Key words: biomarker, microRNA, peripheral blood, gastric cancer, prognosis

\section{Introduction}

Gastric cancer is the second leading cause of cancer-related death globally (1). The incidence of gastric cancer and the mortality rate have been increasing rapidly each year. Recently, the tissue- and disease-specific expression profiles of microRNAs (miRNAs) have attracted considerable attention. MiRNAs are a class of non-coding short RNA molecules (22-24 nucleotides in length) that regulate gene expression at the post-transcriptional level and function as oncogenes or tumor suppressors in human malignancies (2-4). Accumulated evidence indicates that miRNAs are associated with gastric cancer progression and prognosis. Xiao et al reported on the clinical significance of miR-106a in gastric cancer and found that its expression in tissues was associated with tumor stage, size, differentiation, invasion and metastasis (5). Ueda et al reported the results of miRNA expression profiling in gastric cancer tissues and demonstrated that elevated levels of let-7 and miR-214 are associated with an unfavorable outcome (6). Li et al developed a seven-miRNA signature that functions as an independent predictor for relapse-free and overall survival in patients with gastric cancer (7).

Recently, several studies have demonstrated that dysregulated miRNAs originating from tumor tissues are stable and easily detected in the serum or plasma of cancer patients. Since Mitchell et al first investigated circulating miRNAs in prostate cancer, tumor-derived miRNAs in circulation have become an emerging and interesting area of research into the exploitation of minimally invasive biomarkers for cancer detection and prediction (8). In gastric cancer, Tsujiura et al compared several miRNA expression levels between pre-operative and post-operative plasma groups and evaluated four miRNAs (miR-106a, miR-106b, miR-17-5p and let-7) for diagnostic application (9). Concurrently, Zhou et al determined the concentration of circulating miR-106a and miR-17 between gastric cancer patients and healthy controls (10). Both research groups demonstrated that miR-106a and miR-17 serve as a potential diagnostic marker for gastric cancer. Recently, Liu et al performed solexa sequencing to identify differential miRNAs in serum, and created a five-miRNA profile (miR-1, miR-20a, miR-27a, miR-34 and miR-423-5p) for gastric cancer diagnosis and indication of progression (11). Over the 
past decade, increasing effort has focused on the exploitation of new sensitive and specific markers for cancer diagnosis. However, to date, no study has been reported on the prognosis and monitoring of therapeutic response via circulating miRNAs in gastric cancer.

Our previous work (unpublished data) demonstrated that miR-17-5p/20a (miR-17-5p and miR-20a) act as oncogenes and favor gastric cancer cell growth. Knockdown of miR17-5p/20a significantly decreased gastric cancer cell-derived xenograft growth in a mouse model in vivo. In the present study, we initially determined the levels of circulating miR$17-5 \mathrm{p} / 20 \mathrm{a}$ in plasma of gastric cancer patients in an unpaired pre-operative group and assessed the clinical significance. We then performed a comparison of the levels of plasma circulating $\mathrm{miR}-17-5 \mathrm{p} / 20 \mathrm{a}$ among the pre-operative, post-operative, and relapse groups. Additionally, we analyzed the application of prognostic prediction in the unpaired pre-operative group. Finally, we tested the changes in the circulating miR-17-5p/20a levels in tumor-bearing nude mice treated with antagomir-17$5 \mathrm{p} / 20 \mathrm{a}$ and investigated the roles of the two miRNAs in the monitoring of chemotherapeutic effects.

\section{Materials and methods}

Patients and plasma samples. A volume of $2 \mathrm{ml}$ of anticogulated blood was collected from all subjects. The patients had received no previous treatment prior to surgery. Unpaired pre-operative blood samples were collected from 65 patients with gastric cancer, post-operative blood samples were collected from 16 patients one-week after surgery, and the relapse group blood samples were collected from six patients at the Department of Surgery, The First People's Hospital of Zhenjiang. The detailed clinicopathological characteristics of these patients are shown in Table I. The paired pre-operative and post-operative blood samples from 14 patients with gastric cancer were provided by the Department of Sample Bank, Nantong Tumor Hospital. Survival was calculated for the unpaired pre-operative group as months from the date of diagnosis to the date of death or censoring as of April 20, 2011. Survival data were available for 61 of the 65 patients. Informed consent was obtained from all subjects, and the Ethics Committee of Jiangsu University approved all aspects of the study.

The collected blood samples were centrifuged at $3000 \mathrm{rpm}$ for $15 \mathrm{~min}$. The upper plasma was then transferred into a new tube and centrifuged at $12000 \mathrm{x}$ g for $10 \mathrm{~min}$ to remove cellular fractions. Finally, the isolated plasma was aliquoted and stored at $-80^{\circ} \mathrm{C}$ until use.

RNA isolation and real-time $R T-P C R$. Total RNA was extracted from $400 \mu \mathrm{l}$ of plasma using Axyprep ${ }^{\mathrm{TM}}$ multisource total RNA miniprep kit (Axygen Scientific, Inc., Union City, CA, USA) and finally prepared in $15 \mu \mathrm{l}$ of RNase-free water according to the manufacturer's protocol. RNA was reverse transcribed by the specific stem-loop primers for the miRNA assay with RevertAid First Strand cDNA Synthesis kit (Fermentas, Inc., Glen Burnie, MD, USA). Real-time RT-PCR was performed using the BioEasy SYBR-Green I real-time PCR kit (Hangzhou Bioer Techonology Co., Ltd., Hangzhou, China) and the Rotor-6000 real-time fluorescence thermal cycler (Corbett Life Science/Qiagen, Doncaster, Victoria,
Table I. Clinicopathological characteristics in patients with gastric cancer.

\begin{tabular}{|c|c|c|c|}
\hline Parameter & $\begin{array}{c}\text { Pre-operative } \\
\text { group }(\mathrm{n}=65) \\
\mathrm{N}(\%)\end{array}$ & $\begin{array}{c}\text { Post-operative } \\
\text { group }(\mathrm{n}=16) \\
\mathrm{N}(\%)\end{array}$ & $\begin{array}{c}\text { Relapse } \\
\text { group }(n=6) \\
N(\%)\end{array}$ \\
\hline \multicolumn{4}{|l|}{ Gender } \\
\hline Male & $43(66)$ & $11(69)$ & $5 \quad(83)$ \\
\hline Female & $22(34)$ & $5(31)$ & 1 (17) \\
\hline \multicolumn{4}{|l|}{ Age (years) } \\
\hline$\geq 60$ & $44(68)$ & $10(63)$ & $6(100)$ \\
\hline$<60$ & $21(32)$ & $6(37)$ & $0 \quad(0)$ \\
\hline \multicolumn{4}{|c|}{ Tumor diameter (cm) } \\
\hline$\geq 5$ & $33(51)$ & 7 (44) & $4 \quad(67)$ \\
\hline$<5$ & $32(49)$ & $9(56)$ & $2(33)$ \\
\hline \multicolumn{4}{|c|}{ Differentiation } \\
\hline Poor & $36(55)$ & $10(63)$ & $5 \quad(83)$ \\
\hline Moderate & $27(42)$ & $5(31)$ & $1 \quad(17)$ \\
\hline Well & $2(2)$ & $1 \quad(6)$ & $0 \quad(0)$ \\
\hline \multicolumn{4}{|l|}{ Metastasis } \\
\hline Yes & $37(57)$ & $12(75)$ & $6(100)$ \\
\hline No & $28(43)$ & $4(25)$ & $0 \quad(0)$ \\
\hline \multicolumn{4}{|l|}{ TNM stages } \\
\hline $\mathrm{I}+\mathrm{II}$ & $33(51)$ & $7(44)$ & $0 \quad(0)$ \\
\hline III & $24(37)$ & $8(50)$ & $5(83)$ \\
\hline IV & $8(12)$ & $1 \quad(6)$ & $1 \quad(17)$ \\
\hline
\end{tabular}

Australia) for quantitative miRNA detection. The reactions were incubated at $95^{\circ} \mathrm{C}$ for $15 \mathrm{~min}$, followed by 40 cycles of $95^{\circ} \mathrm{C}$ for $15 \mathrm{sec}$ and $60^{\circ} \mathrm{C}$ for $1 \mathrm{~min}$. To obtain a standard curve, the PCR products of the miRNAs and RNU6B (21), a commonly utilized internal control for the miRNA quantification assay, were subcloned into a pMD18-T (Takara, Japan) vector according to the manufacturer's instructions. After serial dilution, standard data were analyzed by real-time analytical software. To ensure specificity of the amplicons, melting curve analysis of the PCR products was performed. Expression levels of miR-17-5p/20a were normalized to U6. All of the primer sequences are listed in Table II.

Cell culture. SGC-7901 cell lines kept in our laboratory were cultured in Dulbecco's modified Eagle's medium supplemented with $10 \%$ fetal bovine serum (Gibco/Invitrogen, Carlsbad, CA, USA) and incubated at $37^{\circ} \mathrm{C}$ in humidified air with $5 \% \mathrm{CO}_{2}$.

Antagomir treatment in xenograft experiment. Four to fiveweek-old BALB/c nude mice were purchased from Slac Laboratory Animal Co.,Ltd.(Shanghai, China).Xenografts were established via subcutaneous injection of $1 \times 10^{6}$ SGC-7901 cells per mouse into the right flank. When the tumor was generated and reached an average volume of $100 \mathrm{~mm}^{3}$, the tumor-bearing nude mice were treated with different reagents, including PBS, antagomir control (ID miR03101), antagomir-17-5p (ID miR31071170508), antagomir-20a (ID miR31071170558) 
Table II. Primer sequences for real-time RT-PCR.

\begin{tabular}{|c|c|c|}
\hline Genes & Primers sequences (5'-3') & Product length (bp) \\
\hline miR-17-5p & $\begin{array}{c}\text { RT: GTCGTATCCAGTGCAGGGTCCGAGGT } \\
\text { ATTCGCACTGGATACGACACTACC } \\
\text { For: GCCGCAAAGTGCTTACAGTG } \\
\text { Rev: TGCAGGGTCCGAGGTAT }\end{array}$ & 61 \\
\hline miR-20a & $\begin{array}{l}\text { RT: GTCGTATCCAGTGCAGGGTCCGAGGT } \\
\text { ATTCGCACTGGATACGACTACCTG } \\
\text { For: GCGGCGGTAAAGTGCTTATAGTG } \\
\text { Rev: TGCAGGGTCCGAGGTAT }\end{array}$ & 62 \\
\hline U6 & $\begin{array}{l}\text { For: CTCGCTTCGGCAGCACA } \\
\text { Rev: AACGCTTCACGAATTTGCGT }\end{array}$ & 94 \\
\hline \multicolumn{3}{|c|}{ RT, reverse transcriptional primer; For, forward primer; Rev, reverse primer. } \\
\hline \multicolumn{3}{|c|}{ Table III. Sequences and modification of oligonucleotides. } \\
\hline Oligonucleotide & Sequences $\left(5^{\prime}-3^{\prime}\right)$ & Modification \\
\hline Antagomir control & $\begin{array}{l}\text { mC(s)mA(s)mGmUmAmCmUmUmUmUmGmUmGmUmAmGmU } \\
\text { mAmC(s)mA(s)mA(s)mA(s)-Chol }\end{array}$ & $\begin{array}{l}\text { 'm' represents 2'-O-methyl- } \\
\text { modified oligonucleotides }\end{array}$ \\
\hline Antagomir-17-5p & $\begin{array}{l}\mathrm{mC}(\mathrm{s}) \mathrm{mU}(\mathrm{s}) \mathrm{mAmCmCmUmGmCmAmCmUmGmUm} \mathrm{AmAmGmCm} \\
\mathrm{AmCmU}(\mathrm{s}) \mathrm{mU}(\mathrm{s}) \mathrm{mU}(\mathrm{s}) \mathrm{mG}(\mathrm{s})-\mathrm{Chol}\end{array}$ & $\begin{array}{l}\text { Subscript 's' represents } \\
\text { phosphorothioate linkage }\end{array}$ \\
\hline Antagomir-20a & $\begin{array}{l}\mathrm{mC}(\mathrm{s}) \mathrm{mU}(\mathrm{s}) \mathrm{mAmCmCmUmGmCmAmCmUmAmUm} \mathrm{AmAmGmC} \\
\mathrm{mAmCmU}(\mathrm{s}) \mathrm{mU}(\mathrm{s}) \mathrm{mU}(\mathrm{s}) \mathrm{mA}(\mathrm{s})-\mathrm{Chol}\end{array}$ & $\begin{array}{l}\text { 'Chol' represents } \\
\text { linked cholesterol }\end{array}$ \\
\hline
\end{tabular}

(Guangzhou RiboBio Co., Ltd., Guangzhou, China) and antagomir-17+20a (antagomir-17-5p and antagomir-20a) $(n=3$ for each reagents). The mice received intratumoral injections of $100 \mu \mathrm{l}$ of antagomir control and antagomir (diluted in PBS at $25 \mu \mathrm{mol} / \mathrm{l}$ ) and an equal volume of PBS twice per week for 2 weeks. The animal studies were performed in accordance with the guidelines of the Institutional Animal Care and Use Committee of Jiangsu University. Sequences and modifications of the antagomirs are shown in Table III.

When the volume of xenografts reached $100 \mathrm{~mm}^{3}$, serum from the pre-treatment mice was collected. After xenografts were treated with different reagents, serum from the posttreatment mice was obtained at 10 and 20 days post-treatment, respectively. All the serum samples were prepared according to the above process of plasma preparation.

Statistical analysis. Experiments were repeated three times. Statistical analysis was performed with SPSS 17.0 and GraphPad Prism 5. The Mann-Whitney U test, Kruskal-Wallis test or one-way ANOVA was used to assess differences in different groups. The Wilcoxon signed rank test was performed to determine the significance of the difference of circulating miRNA levels between the paired pre-operative and postoperative plasma groups. Survival curves were constructed using the Kaplan-Meier method and survival differences were assessed using the Log-rank test. Univariate and multivariate analyses of the prognostic factors were performed using the
Cox proportional hazards model. A probability level of $\mathrm{P}<0.05$ was chosen for statistical significance.

\section{Results}

Clinical significance of the circulating $\mathrm{miR}-17-5 \mathrm{p} / 20$ a levels in the pre-operative plasma group. Initially, we determined the circulating microRNA (miR-17-5p/20a) levels in the plasma of 65 patients prior to surgery; median values for the circulating miR-17-5p and miR-20a were 0.152 and 0.260 , respectively. According to the median values, we divided the pre-operative plasma into low expression and high expression groups in combination with the clinicopathological data (Table IV). Next, we compared the concentrations of the circulating miR-17-5p/20a among different clinicopathological parameter groups and assessed the clinical significance. As indicated in Table IV and Fig. 1, the levels of miR-17-5p and miR-20a were significantly associated with the differentiation status and TNM staging of gastric cancer. The concentrations of circulating miRNAs in the group exhibiting poor differentiation were higher than that of the moderate differentiation group $(\mathrm{P}=0.018$ for $\mathrm{miR}-17-5 \mathrm{p}$ and $\mathrm{P}=0.005$ for $\mathrm{miR}=20 \mathrm{a}$ ), whereas there were no differences noted between the well differentiation group and the poor or moderate differentiation group (Fig. 1A and B). Our data indicated that the concentrations of circulating miRNAs in the TNM III stage group were significantly higher than that in the TNM I+II group (TNM I and 
Table IV. Clinicopathological correlation of plasma miR-17-5p/20a expression levels in pre-operative group.

\begin{tabular}{|c|c|c|c|c|c|c|}
\hline \multirow[b]{2}{*}{ Parameter } & \multicolumn{3}{|c|}{ miR-17-5p expression } & \multicolumn{3}{|c|}{ miR-20a expression } \\
\hline & Low & High & P-value & Low & High & P-value \\
\hline \multicolumn{7}{|l|}{ Gender } \\
\hline Male & 21 & 22 & \multirow{2}{*}{$0.790^{\mathrm{a}}$} & 21 & 22 & \multirow[t]{2}{*}{$0.323^{\mathrm{a}}$} \\
\hline Female & 11 & 11 & & 10 & 12 & \\
\hline \multicolumn{7}{|l|}{ Age (years) } \\
\hline$\geq 60$ & 22 & 22 & \multirow[t]{2}{*}{$0.884^{\mathrm{a}}$} & 18 & 26 & \multirow[t]{2}{*}{$0.647^{\mathrm{a}}$} \\
\hline$<60$ & 10 & 11 & & 13 & 8 & \\
\hline \multicolumn{7}{|c|}{ Tumor diameter $(\mathrm{cm})$} \\
\hline$\geq 5$ & 14 & 19 & \multirow[t]{2}{*}{$0.798^{a}$} & 14 & 19 & \multirow[t]{2}{*}{$0.582^{\mathrm{a}}$} \\
\hline$<5$ & 18 & 14 & & 17 & 15 & \\
\hline \multicolumn{7}{|c|}{ Differentiation } \\
\hline Poor & 14 & 22 & \multirow[t]{3}{*}{$0.039^{\mathrm{b}, \mathrm{c}}$} & 12 & 24 & \multirow[t]{3}{*}{$0.015^{\mathrm{b}, \mathrm{c}}$} \\
\hline Moderate & 18 & 9 & & 18 & 9 & \\
\hline Well & 0 & 2 & & 1 & 1 & \\
\hline \multicolumn{7}{|l|}{ Metastasis } \\
\hline Yes & 15 & 22 & \multirow[t]{2}{*}{$0.393^{\mathrm{a}}$} & 15 & 22 & \multirow[t]{2}{*}{$0.337^{\mathrm{a}}$} \\
\hline No & 17 & 11 & & 16 & 12 & \\
\hline \multicolumn{7}{|l|}{ TNM stages } \\
\hline $\mathrm{I}+\mathrm{II}$ & 15 & 8 & \multirow[t]{3}{*}{$0.001^{\mathrm{b}-\mathrm{d}}$} & 14 & 9 & \multirow[t]{3}{*}{$0.001^{\mathrm{b}-\mathrm{d}}$} \\
\hline III & 14 & 20 & & 3 & 21 & \\
\hline IV & 3 & 5 & & 4 & 4 & \\
\hline
\end{tabular}

${ }^{\mathrm{a}}$ Mann-Whitney U test; ${ }^{\mathrm{b}} \mathrm{Kruskal-Wallis} \mathrm{test;}{ }^{\mathrm{c}} \mathrm{P}<0.05$; ${ }^{\mathrm{d}}$ indicates the data are $<0.001$.

II stages) ( $\mathrm{P}<0.001$ for miR-17-5p and miR-20a, respectively). No significant differences were detected between the TNM IV stage group and the TNM I+II or III stage group (Fig. 1C and D).

Changes in the circulating miR-17-5p/20a levels in different plasma groups. We performed real-time RT-PCR to measure and compare the circulating expression levels in the paired pre-operative and post-operative plasma samples $(n=14)$. As we hypothesized, the levels of the circulating miRNAs were notably reduced in the post-operative group $(\mathrm{P}<0.001$ for miR-17-5p and miR-20a, respectively) (Fig. 2A and B). These results indicate that the elevated levels of the circulating miR$17-5 \mathrm{p} / 20 \mathrm{a}$ in the pre-operative plasma were potentially due to the release by gastric cancer cells and the elevated levels are potentially useful for detection of gastric cancer. The recurrence of gastric cancer may exhibit elevated circulating miRNA levels. Therefore, we collected unpaired pre-operative $(n=65)$, post-operative $(n=16)$, and the relapse $(n=6)$ plasma samples and analyzed the changes in the circulating $\mathrm{miR}-17-5 \mathrm{p} / 20 \mathrm{a}$ among the three groups. Interestingly, the levels of miRNAs were indeed significantly reduced in the post-operative group $(\mathrm{P}<0.001$ for both miR-17-5p and miR-20a) and only the level of miR-17-5p in the relapse group was significantly increased and exhibited a similar level to that in the pre-operative group $(\mathrm{P}=0.003$ for miR-17-5p) (Fig. 2C). The concentration of miR-20a in the relapse group was erratic, but no significance
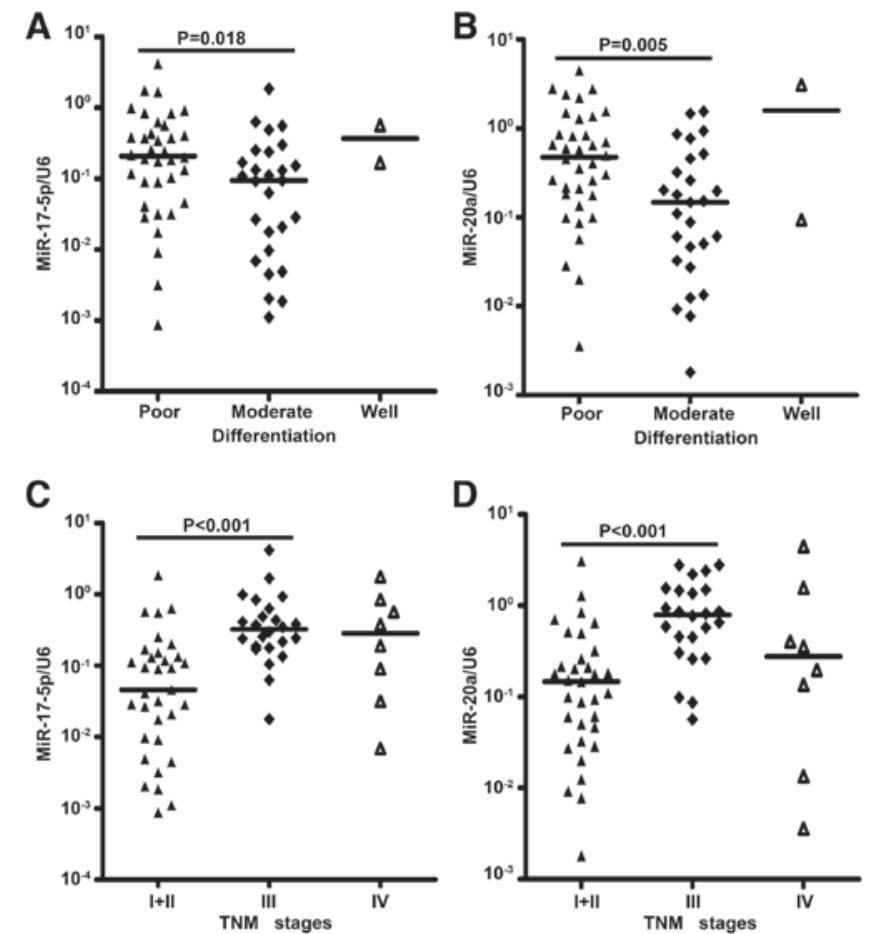

Figure 1. Clinical significance of the plasma miR-17-5p/20a levels in gastric cancer. The unpaired pre-operative patients were recruited for assay. (A and B) Scatter plot of the circulating miRNA expression levels in the poor, moderate and well differentiation groups. (C and D) Scatter plot of the circulating miRNA expression levels in different TNM stages. I+II, TNM I and TNM II stages. 

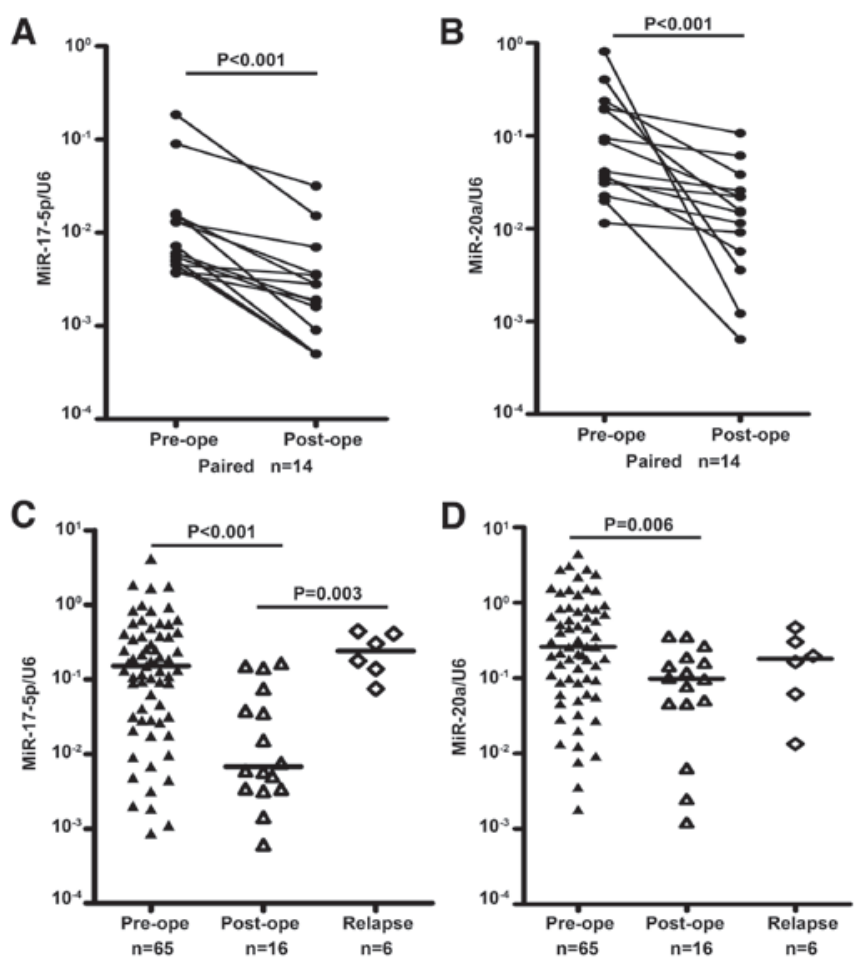

Figure 2. Fluctuation of the circulating miR-17-5p/20a expression in different plasma groups. (A and B) Comparison of miRNA concentrations between the paired pre-operative and post-operative plasma $(n=14)$. The levels of miR-17$5 \mathrm{p} / 20$ a are significantly reduced in plasma samples obtained one week after surgical removal of gastric cancer. (C and D) Scatter plot of miRNA levels in the pre-operative $(n=65)$, post-operative $(n=16)$ and relapse $(n=6)$ plasma groups.

was detected when compared to the post-operative group (Fig. 2D). These findings indicated that the circulating levels of miR-17-5p/20a might reflect the burden of gastric cancer and pathological progression.

Predictive power of circulating miR-17-5p/20a levels for gastric cancer prognosis. To achieve a better understanding of the potential roles of the circulating miR-17-5p/20a regarding a prognostic application for gastric cancer, we investigated the association between the circulating miR-17-5p/20a levels with overall survival. A comparison of survival rates between low expression and high expression groups was conducted. Analysis using a Kaplan-Meier curve demonstrated that patients with higher expression levels of the circulating miR$17-5 \mathrm{p} / 20 \mathrm{a}$ had a dramatically lower survival rate compared to the low expression group ( $\mathrm{P}=0.003$ for both miR-17-5p and miR-20a) (Fig. 3).

We utilized univariate and multivariate Cox regression models to identify the variables of potential prognostic significance in all gastric cancer patients. Univariate Cox regression analysis indicated that the elevated plasma miR-17-5p/20a levels were significantly correlated with overall survival $(\mathrm{P}=0.017$ for miR-17-5p and $\mathrm{P}<0.001$ for miR-20a) (Table V). The univariate Cox regression analysis also indicated that clinicopathological characteristics, including differentiation status and TNM stage, were significantly associated with overall survival $(\mathrm{P}=0.013$ and $\mathrm{P}=0.002$, respectively) (Table $\mathrm{V})$.
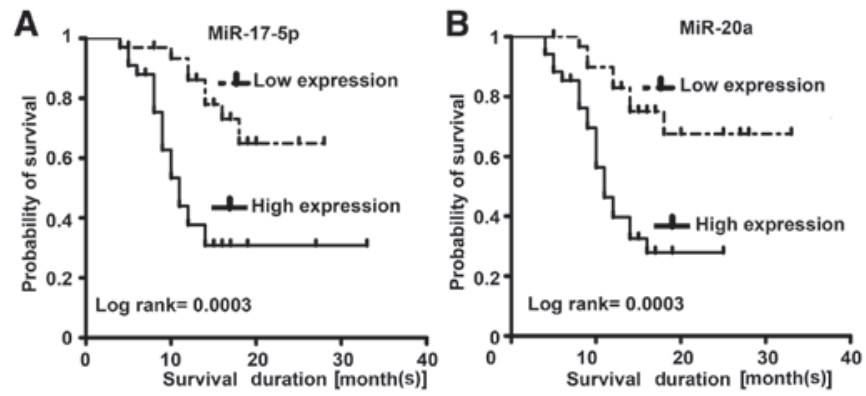

Figure 3. Kaplan-Meier analysis of overall survival based on the levels of the circulating miR-17-5p/20a in the pre-operative plasma group. The expression levels of miRNAs are dichotomized relative to the median value: low expression group and higher expression group. (A) Kaplan-Meier curve for miR-17-5p; (B) Kaplan-Meier curve for miR-20a.
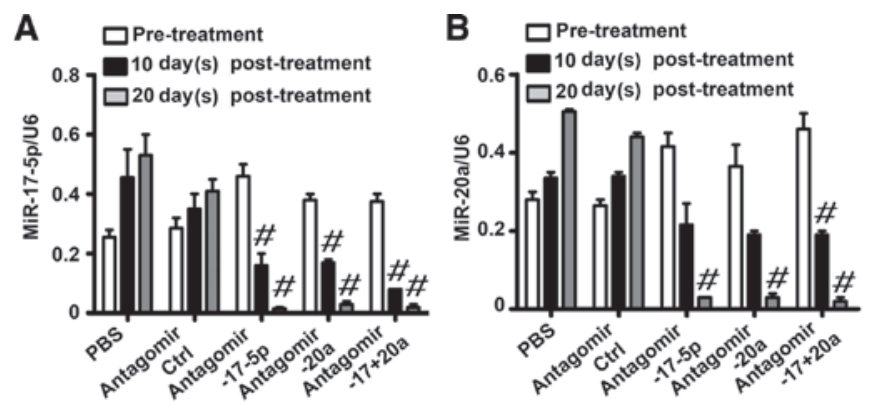

Figure 4. Detection of miR-17-5p/20a in serum from antagomir-treated mice. The changes in the serum miRNA levels after tumor-bearing mice were treated with different reagents: PBS, antagomir control, antagomir-17-5p, antagomir20a, antagomir-17+20a (antagomir-17-5p and antagomir-20a) $(n=3$ for each reagent). (A) miR-17-5p; (B) miR-20a. Data were presented as mean or mean \pm standard deviation (SD). One-way ANOVA was used to assess differences. Difference from the pre-treatment group were noted; ${ }^{\#} \mathrm{P}<0.05$.

To determine whether plasma miR-17-5p/20a levels were independent risk factors for poor prognosis, both clinicopathological factors and the levels of the circulating miR-17-5p/20a were evaluated by multivariate Cox regression along with backward Wald test. The results indicated that TNM stage and an elevated level of plasma miR-20a were both independent risk factors in predicting the overall survival of patients with gastric cancer $(\mathrm{P}=0.029$ and $\mathrm{P}=0.013$, respectively) (Table $\mathrm{V})$.

Changes in the circulating miR-17-5p/20a levels after xenograft-bearing mice were treated with antagomirs. Surgery and chemotherapy are accepted as the two principal therapeutic strategies for gastric cancer patients. The resection of gastric cancer tissues leads to down-regulation of the circulating miR$17-5 \mathrm{p} / 20$ a levels. Whether the circulating miR-17-5p/20a levels could be indicative of the therapeutic effects of chemotherapy warrants further evaluation. Currently, patients treated with chemotherapy alone are rare, thus the acquisition of plasma from these patients is challenging. Our previous research demonstrated that antagomirs against miR-17-5p/20a that are applied as chemotherapeutic reagents significantly inhibit tumor growth in vivo. Antagomirs against miR-17-5p/20a could potentially serve as an alternative to a chemotherapeutic approach; additionally, the circulating miR-17-5p/20a levels 
Table V. Univariate and multivariate analysis for the prognostic significance of clinicopathological characteristics and plasma $\mathrm{miR}-17-5 \mathrm{p} / 20 \mathrm{a}$ levels in gastric cancer.

\begin{tabular}{|c|c|c|c|c|}
\hline \multirow[b]{2}{*}{ Parameter } & \multicolumn{2}{|c|}{ Univariate analysis } & \multicolumn{2}{|c|}{ Multivariate analysis ${ }^{\mathrm{a}}$} \\
\hline & $\operatorname{HR}(95 \% \mathrm{CI})^{\mathrm{b}}$ & P-value & $\mathrm{HR}(95 \% \mathrm{CI})^{\mathrm{b}}$ & P-value \\
\hline Gender $^{\mathrm{c}}$ & $1.354(0.619-2.961)$ & 0.448 & & \\
\hline $\mathrm{Age}^{\mathrm{c}}$ & $1.392(0.619-3.129)$ & 0.424 & & \\
\hline Tumor diameter $^{\mathrm{c}}$ & $1.223(0.596-2.508)$ & 0.583 & & \\
\hline Differentiation $^{c}$ & $2.925(1.253-6.827)$ & $0.013^{\mathrm{d}}$ & & \\
\hline Metastasis $^{\mathrm{c}}$ & $1.625(0.772-3.418)$ & 0.201 & & \\
\hline TNM stages $^{c}$ & $3.396(1.549-7.447)$ & $0.002^{\mathrm{d}}$ & $2.526(1.099-5.803)$ & $0.029^{\mathrm{d}}$ \\
\hline miR-17-5p & $1.785(1.110-2.870)$ & $0.017^{\mathrm{d}}$ & & \\
\hline miR-20a & $1.818(1.321-2.502)$ & $0.000^{\mathrm{d}}$ & $1.576(1.102-2.253)$ & $0.013^{\mathrm{d}}$ \\
\hline
\end{tabular}

${ }^{\mathrm{a} B a c k w a r d}$ Wald test used for variables screened, $\mathrm{P}=0.05$ was chosen as a criteria for significance; ${ }^{\mathrm{b}} \mathrm{CI}, 95 \%$ confidence interval; ${ }^{\mathrm{c}}$ gender, male vs. female; age, $\geq 60$ vs. $<60$ years; tumor diameter, $\geq 5$ vs. $<5 \mathrm{~cm}$; differentiation, poor vs. moderate and well; metastasis, yes vs. no; TNM stage, III and IV vs. I and II; ${ }^{\mathrm{P}}<0.05$.

could potentially be affected after xenograft treatment with antagomirs, thus indicating the therapeutic effects.

To test this hypothesis, we determined and compared the levels of the circulating $\mathrm{miR}-17-5 \mathrm{p} / 20 \mathrm{a}$ in the sera of pretreated mice and the antagomir-treated mice. Compared to the serum miRNAs levels of the pre-treated group, we found that the circulating levels of miR-17-5p/20a were gradually reduced in the antagomir-treated group at 10 and 20 days after treatment (the change in serum miR-17-5p: $\mathrm{P}=0.012$ and $\mathrm{P}=0.004$ for antagomir-17-5p, $\mathrm{P}=0.003$ and $\mathrm{P}=0.001$ for antagomir-20a, $\mathrm{P}=0.001$ and $\mathrm{P}=0.001$ for antagomir $-17+20 \mathrm{a}$; the change in serum miR-20a: $\mathrm{P}=0.054$ and $\mathrm{P}=0.009$ for antagomir-17-5p, $\mathrm{P}=0.053$ and $\mathrm{P}=0.009$ for antagomir-20a, $\mathrm{P}=0.007$ and $\mathrm{P}=0.002$ for antagomir-17+20). However, the levels remained elevated in the PBS and antagomir control groups (Fig. 4).

\section{Discussion}

Accumulating evidence indicates that dysregulated miRNAs originating from cancer cells are stable and easily detected in plasma or serum of patients with cancer (12-14). A great body of research concerning circulating miRNAs in colorectal cancer (15), hepatocarcinoma (16) and breast cancer (17) suggests that circulating miRNAs are potential non-invasive markers for diagnosis and prognotic prediction of cancer $(18,19)$. With respect to gastric cancer, research has been conducted to explore the alteration of circulating miRNAs between gastric cancer patients and healthy controls and to search for diagnostic markers, however, no further investigation has been carried out to date. Therefore, in the present study, we focused on the clinical significance, prognotic predictive power and chemotherapeutic monitoring capabilities of circulating levels of $\mathrm{miR}-17-5 \mathrm{p} / 20 \mathrm{a}$ in gastric cancer.

In investigating the clinical significance of plasma miR$17-5 \mathrm{p} / 20 \mathrm{a}$, we found that plasma $\mathrm{miR}-17-5 \mathrm{p} / 20 \mathrm{a}$ levels were significantly associated with differentiation status and TNM stages. Notably, poorly differentiation and TNM III stage both exhibited the highest levels of circulating miR-17-5p/20a among the corresponding groups. Meanwhile, the absence of difference noted in the other two groups may be attributed to the small number of samples for assay. Large number of highgrade patients need to be included for analysis. In addition, the clinical significance of circulating levels of $\mathrm{miR}-17-5 \mathrm{p} / 20 \mathrm{a}$ was found to differ from the results of the tissue-based assays of our previous research, which indicates that the level of miRNAs in cancer tissues may not represent the profiles of the corresponding miRNA levels in plasma (20). The application of dysregulated miRNAs in gastric cancer tissue warrants further evaluation in comparison to the circulating form.

Comparison of the circulating miR-17-5p/20a levels between the pre-operative and post-operative plasma groups indicated that the levels in the post-operative groups were both significantly decreased, regardless of whether the samples were paired plasma from the same patient or unpaired plasma, a result consistent with a report by Tsujiura et al (9). The decrease in the circulating miRNA levels in the post-operative plasma may indicate that plasma $\mathrm{miR}-17-5 \mathrm{p} / 20 \mathrm{a}$ are released by gastric cancer cells. The concentration of the circulating miRNAs could potentially be used to test for recurrence in patients after operation. In order to confirm this hypothesis, we also collected plasma from relapsed gastric cancer patients, and tested the levels of circulating miR-17-5p/20a. The levels of $\mathrm{miR}-17-5 \mathrm{p} / 20 \mathrm{a}$ in this group were elevated and reached the levels in the plasma of the pre-operative patients. These results further indicate that cancer-associated miRNAs may be released by cancer cells into the peripheral blood and may be explored as useful biomarkers.

In order to investigate potential roles of the circulating miR-17-5p/20a in prognotic prediction, we conducted a 3-year survival follow-up for unpaired pre-operative patients and found that patients with higher levels of circulating miR17-5p/20a exhibit lower survival rates. The univariate and multivariate analysis with Cox regression models revealed that only miR-20a potentially serves as an independent risk 
predictor for the prognosis of gastric cancer, while miR-17-5p was not. Although these two miRNAs are produced and function in the same way, differences between them exist. The above data showed that the circulating levels of miR-17-5p were more associated with pathological progression of gastric cancer than miR-20a, which may give an explanation that miR$17-5 p$ could not be analyzed as an independent predictor for prognosis of gastric cancer.

In addition, we established a mouse tumor model and conducted chemotherapy in vivo. We analyzed the role of circulating levels of miR-17-5p/20a in the monitoring of chemotherapeutic effects. When antagomirs against miR-17-5p/20a were used, tumor growth was obviously restricted and the circulating miRNA levels gradually decreased. But their levels were still elevated in PBS- and antagomir control-treated groups. These implied that the circulating miR-17-5p/20a levels may be effected by burden of tumor and potentially be used to monitor the therapeutic response and effect in patients. However, the concentration of the levels of miR-17-5p/20a in plasma from a large number of patients treated with chemotherapeutic drugs needs to be further evaluated. Moreover, other miRNAs involved in gastric cancer tissues could be considered and measured to assess their potential application in circulating form.

In conclusion, our findings suggest that the circulating miR$17-5 \mathrm{p} / 20 \mathrm{a}$ levels in plasma are associated with pathological progression of gastric cancer and serve as a non-invasive biomarker for the evaluation of therapeutic effects and the prediction of gastric cancer patient prognosis.

\section{Acknowledgements}

We thank the International Science Editing Co. for language polishing and grammatical editing. This study was supported by the National Natural Science Foundation of China (grant no. 81071421), the Natural Science Foundation of the Jiangsu Province (grant nos. BK2007705 and 2009055), the Sci-Tech innovation team and talents of Jiangsu University (grant no. 2008-018-02). Jiangsu Province Doctoral Innovation Fund (Grant no. CXZZ11_0592).

\section{References}

1. Parkin DM, Bray F, Ferlay J and Pisani P: Global cancer statistics, 2002. CA Cancer J Clin 55: 74-108, 2005.

2. Bartel DP: MicroRNAs: genomics, biogenesis, mechanism, and function. Cell 116: 281-297, 2004.
3. Fabian MR, Sonenberg N and Filipowicz W: Regulation of mRNA translation and stability by microRNAs. Annu Rev Biochem 79: 351-379, 2010.

4. Calin GA and Croce CM: MicroRNA signatures in human cancers. Nat Rev Cancer 6: 857-866, 2006.

5. Xiao B, Guo J, Miao Y, et al: Detection of miR-106a in gastric carcinoma and its clinical significance. Clin Chim Acta 400: 97-102, 2009

6. Ueda T, Volinia S, Okumura H, et al: Relation between microRNA expression and progression and prognosis of gastric cancer: a microRNA expression analysis. Lancet Oncol 11: 136-146, 2010

7. Li X, Zhang Y, Ding J, Wu K and Fan D: Survival prediction of gastric cancer by a seven-microRNA signature. Gut 59: 579-585, 2010.

8. Mitchell PS, Parkin RK, Kroh EM, et al: Circulating microRNAs as stable blood-based markers for cancer detection. Proc Natl Acad Sci USA 105: 10513-10518, 2008.

9. Tsujiura M,Ichikawa D, Komatsu S, et al: Circulating microRNAs in plasma of patients with gastric cancers. Br J Cancer 102: 1174-1179, 2010.

10. Zhou H, Guo JM, Lou YR, et al: Detection of circulating tumor cells in peripheral blood from patients with gastric cancer using microRNA as a marker. J Mol Med (Berl) 88: 709-717, 2010.

11. Liu R, Zhang $\mathrm{C}, \mathrm{Hu} \mathrm{Z}$, et al: A five-microRNA signature identified from genome-wide serum microRNA expression profiling serves as a fingerprint for gastric cancer diagnosis. Eur J Cancer 47: 784-791, 2011.

12. Mostert B, Sieuwerts AM, Martens JW and Sleijfer S: Diagnostic applications of cell-free and circulating tumor cell-associated miRNAs in cancer patients. Expert Rev Mol Diagn 11: 259-275, 2011.

13. Brase JC, Wuttig D, Kuner R and Sultmann H: Serum microRNAs as non-invasive biomarkers for cancer. Mol Cancer 9: 306, 2010.

14. Zen K and Zhang CY: Circulating MicroRNAs: a novel class of biomarkers to diagnose and monitor human cancers. Med Res Rev: Nov 9, 2010 (Epub ahead of print).

15. Ng EK, Chong WW, Jin H, et al: Differential expression of microRNAs in plasma of patients with colorectal cancer: a potential marker for colorectal cancer screening. Gut 58: 1375-1381, 2009.

16. Li LM, Hu ZB, Zhou ZX, et al: Serum microRNA profiles serve as novel biomarkers for HBV infection and diagnosis of HBV-positive hepatocarcinoma. Cancer Res 70: 9798-9807, 2010.

17. Zhao H, Shen J, Medico L, Wang D, Ambrosone CB and Liu S: A pilot study of circulating miRNAs as potential biomarkers of early stage breast cancer. PLoS One 5: e13735, 2010.

18. Kosaka N, Iguchi $\mathrm{H}$ and Ochiya T: Circulating microRNA in body fluid: a new potential biomarker for cancer diagnosis and prognosis. Cancer Sci 101: 2087-2092, 2010.

19. Ferracin M, Veronese A and Negrini M: Micromarkers: miRNAs in cancer diagnosis and prognosis. Expert Rev Mol Diagn 10: 297-308, 2010.

20. Rosell R, Wei J and Taron M: Circulating microRNA signatures of tumor-derived exosomes for early diagnosis of non-small cell lung cancer. Clin Lung Cancer 10: 8-9, 2009.

21. Chen C, Ridzon DA, Broomer AJ, et al: Real-time quantification of microRNAs by stem-loop RT-PCR. Nucleic Acids Res 33: e179, 2005. 